\title{
CARIBENA DE
}

CIENCIAS SOCIALES

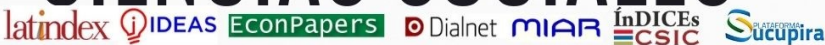

\section{MÉTODO MIXTO DE INVESTIGACIÓN: PERTINENCIA Y DIFICULTADES EN EL ESTUDIO PROGRAMAS DE REENGANCHE}

\author{
María Trinidad Cutanda-López \\ Profesora Ayudante Doctor. Universidad de Murcia \\ https://orcid.org/0000-0003-0246-9382 \\ Lopez.cutanda@um.es \\ Correspondencia: lopez.cutanda@um.es
}

Para citar este artículo puede utilizar el siguiente formato:

María Trinidad Cutanda-López: "Método mixto de investigación: pertinencia y dificultades en el estudio programas de reenganche", Revista Caribeña de Ciencias Sociales (Especial noviembre 2021, pp. 3148) En línea:

https://doi.org/10.51896/caribe/MGUI5478

\section{RESUMEN}

Se aportan algunas consideraciones sobre la pertinencia del Método Mixto de Investigación (MMI) para el análisis de programas de re-enganche. En particular, las Aulas Ocupacionales en la Región de Murcia (España). A raíz de la revisión bibliográfica, legislativa y metodológica realizada en el contexto de una Tesis Doctoral ${ }^{1}$, se parte de posicionamientos críticos teórico-políticos sobre el empleo predominante de estudios cuantitativos para estudiar problemáticas como fracaso, abandono o desenganche escolar, que pueden proporcionar repuestas simplistas limitando la concreción de metas y responsabilidades. Se sugiere el empleo de enfoques más profundos y flexibles (cualitativos) que, junto a las cifras, permitan dar voz y conocer las vivencias de sus implicados. Los resultados, de una parte, justifican el MMI como una tercera vía de indagación (junto al enfoque cuantitativo y cualitativo) en la investigación social y educativa; aporta, desde una mirada más flexible, funcional, conciliadora y enriquecida, nuevas formas de aproximación y solución. De otro, evidencian diversas dificultades: necesidad de justificar y explicitar su adecuación para mitigar susceptibles cuestionamientos; mayor tiempo y recursos; entrenamiento, conocimientos y habilidades en métodos cuantitativos y cualitativos; diferentes ritmos y tiempos de indagación. Las conclusiones subrayan la importancia y pertinencia del MMI en el estudio de realidades y problemáticas educativas de gran complejidad como las analizadas en el marco del Aula Ocupacional, permitiendo indagar en sus múltiples planos y condicionantes de forma interconectada, en consonancia con los posicionamientos

\footnotetext{
${ }^{1}$ Tesis Doctoral (2019), financiada por el Ministerio de Economía y Competitividad (BES-2013-063943), "La implicación del alumnado absentista y en riesgo de abandono en su propio aprendizaje y el papel de la formación, el compromiso y el trabajo en el aula de los docentes. El caso de las Aulas Ocupacionales en la Región de Murcia"
} 
teóricos de partida (enfoque ecológico del riesgo escolar y enfoque multidimensional de School Engagement).

Palabras clave: Aulas Ocupacionales; Programas de Re-enganche; Método Mixto de Investigación; School Engagement; Vulnerabilidad escolar.

\section{MIXED RESEARCH METHOD: RELEVANCE AND DIFFICULTIES IN RESEARCH ON REENGAGEMENT PROGRAMS}

\section{ABSTRACT}

Some considerations are provided on the relevance of the Mixed Method Research (MMR) for the analysis of reengagement programs. In particular, Aulas Ocupacionales in the Region of Murcia (Spain). As a result of the bibliographic, legislative and methodological review carried out in the context of a Doctoral Thesis, it is based on theoretical-political critical positions on the predominant use of quantitative studies to study problems such as failure, dropout or school disengagement, which can provide simplistic answers limiting the realization of goals and responsibilities. The use of deeper and more flexible (qualitative) approaches is suggested that, together with the figures, give voice and know the experiences of those involved. The results, on the one hand, justify the MMR as a third way of inquiry (together with the quantitative and qualitative approach) in social and educational research; It provides, from a more flexible, functional, conciliatory and enriched perspective, new forms of approach and solution. On the other, they show several difficulties: the need to justify and make explicit their adequacy to mitigate susceptible questions; increased time and resources; training, knowledge and skills in quantitative and qualitative methods; different rhythms and times of inquiry. The conclusions underline the importance and relevance of the MMR in the study of realities and educational problems of great complexity such as those analyzed in the framework of Aula Ocupacional, allowing to investigate its multiple planes and conditions in an interconnected way, in line with the theoretical positions of departure (ecological approach to school risk and multidimensional approach of School Engagement).

Keywords: Aula Ocupacional; Mixed Methods Research; Re.-engagement Programs; School Engagement; School Vulnerability.

\section{INTRODUCCIÓN}

Fracaso y abandono escolar y, los procesos de desvinculación que los propician, continúan a día de hoy como uno de los mayores y más perseverantes retos políticos, sociales y educativos. Más allá de la visibilidad y publicidad de sus cifras y a pesar de los grandes propósitos y lemas inclusivos, persisten en la vida de muchos estudiantes como una epidemia silenciosa (Rumberger \& Rotermund, 2012, p. 491). La preocupación por estos fenómenos y sus importantes repercusiones, es notoria en los gobiernos y los ámbitos de la política internacional (OCDE 2016). Y, asimismo, desde la investigación (Day, 2013; GHT Consluting Lt; Nevala \& Hawley, 2011; Marcelo; Araújo; Magalhães y Rocha, 2015; NESSE, 2010, 2011; Pradós, Duque y Molina, 2011; Tomeszewska-Pekata, Marchlik \& 
Wrona, 2017), que ha abordado dichas problemáticas desde múltiples disciplinas, planos y perspectivas diferenciadas (psicológicas, sociológicas, económicas, pedagógicas, curriculares, organizativas, de desarrollo profesional docente...) en consonancia con la complejidad inherente a tales fenómenos y la dificultad para definirlos y comprenderlos en profundidad (Lara-García, González-Palacios, González-Álvarez, Martínez-González, 2014). Se han logrado así avances significativos en la comprensión de tales problemáticas (condiciones, factores, procesos, implicaciones políticas, sociales y escolares...) aunque todavía son muchas las cuestiones pendientes y sin resolver.

Más específicamente, cabe señalar que la investigación llevada a cabo bajo el propósito de documentar, analizar e interpretar las cifras arrojadas por informes de organismos como la OCDE, la plataforma estadística comunitaria (Eurostat), o el Instituto Nacional de Estadística de nuestro país (INEE) ha sido prolija. Sobre el tema, se han pronunciado autores como Bolívar y López (2009); Choi y Calero, 2013; Faci (2011); Fernández, Muñoz de Bustillo, Braña y Antón (2010) o Vaquero (2011). Si bien, se han señalado a su vez ciertas limitaciones respecto a las metodologías e instrumentos de análisis sobre el particular. Entre otros, problemas relacionados con las técnicas utilizadas y la mayor o menor representatividad de los resultados, con su difusión y externalización, con la ausencia de un registro unificado de información o, con la propia fiabilidad de los datos (Bolívar y López, 2009; European Commision, 2015; Faci, 2011; Martos, 2016).

La imprecisión conceptual que caracteriza esta área de indagación, así como una interpretación conveniente de los datos según el interés del momento, dejan un amplio margen de ambigüedad que propicia repuestas simplistas, dificulta los avances en la delimitación de su naturaleza y magnitud, y limita las posibilidades de concretar metas y responsabilidades claras y bien definidas (Comisión Europea, 2011; Rambla y Fontdevilla, 2015). Además, el énfasis de los análisis macro para delimitar el perfil del alumnado en situación de riesgo puede, de una parte, conllevar una concepción simplista y fragmentada consistente en atribuir las causas del fracaso y abandono escolar a los propios estudiantes, sus condiciones y características personales, familiares y socioeconómicas. Sin embargo, las realidades de estos jóvenes no solo se deben a variables personales, sino también y en gran medida a otros condicionantes estructurales y sistémicos que las conforman e influyen en ellas de forma determinante. $Y$ de otra, proporcionar una respuesta focalizada en la derivación de este alumnado a programas específicos, como el Aula Ocupacional, que pueden funcionar en la práctica como vías paralelas, estigmatizadores y de segregación (Escudero y Martínez, 2012; Marhuenda y García, 2017). Es más, en algunos casos, se ha documentado que tales alternativas de escolarización pueden consolidarse como dispositivos institucionales naturalizados en los centros escolares para establecer modificaciones estructurales, alteraciones en los procesos de selección escolar, y jerarquías entre alumnos según perfiles y trayectorias (Rujas, 2017).

Bajo este telón de fondo, el presente artículo parte de los presupuestos de que fracaso, abandono o desenganche escolar y los programas de re-enganche expresamente diseñados e implementados para dar respuestas y lograr re-enganchar al alumnado que, en tales situaciones, se encuentra en situación de vulnerabilidad escolar, requieren tomar conciencia de la complejidad que 
los caracteriza. Esto es, precisan de su estudio desde enfoques comprehensivos que permitan conocer y analizar en profundidad sus claves políticas, institucionales, organizativas y pedagógicas, acordes con el propósito de dilucidar en qué medida realmente contribuyen a lograr unos determinados resultados de aprendizaje e implicación de su alumnado. Se sigue así la estela de investigaciones precedentes el ámbito de estudio que aquí concierne, desde las que se reafirma la necesidad de apostar por enfoques más profundos y flexibles (cualitativos), que, junto a las cifras (cuantitativos), permitan dar voz y conocer las propias vivencias de sus principales afectados (Christenson, Reschly \& Wylie, 2012; Fredericks, Blumenfiel \& Parris, 2004; González, 2015; Harris, 2011; Symonds y Hargreaves, 2016). Asimismo, son diversos los metodólogos que reconocen la pertinencia del Método Mixto de investigación (en adelante, MMI) para el estudio de problemas educativos de tal naturaleza y complejidad como el que aquí nos ocupa (Creswell, 2015; HernándezSampieri, Fernández y Baptista, 2014; Merters, Bazeley, Bowleg, Fielding, Maxwel, Molina-Azorín \& Niglas, 2016).

Partiendo de las consideraciones esgrimidas en la introducción, las líneas que siguen se destinan en primer lugar a delimitar las principales coordenadas que, desde el punto de vista teórico delimitan la emergencia, consolidación y caracterización del MMI justificando su pertinencia en la investigación educativa. Un segundo apartado, corresponde a la metodología del estudio en relación a la revisión bibliográfica, legislativa y metodológica realizada. En tercer lugar, se presentan y discuten los resultados relativos al programa analizado, el Aula Ocupacional, y los principales elementos que lo caracterizan según la legislación vigente, para una vez situados pasar a abordar aquellos correspondientes a la pertinencia y dificultades del MMI en el análisis de las mismas y, más específicamente, en torno al diseño de investigación en particular en el que se posicionó la investigación. Un apartado final de conclusiones cierra el artículo.

\section{MARCO TEÓRICO}

\section{1- Enfoque de Métodos Mixtos de Investigación: una aproximación a su emergencia, consolidación y caracterización.}

Desde el punto de vista de la investigación en general, y de la investigación educativa en particular, aunque tradicionalmente el debate contrapuesto entre enfoques cuantitativo y cualitativo ha sido mayoritario, éste se considera actualmente superado, siendo muchos teóricos y especialistas en el tema los que aluden a una reconciliación en la práctica entre los mismos (Diaz, 2014; HernándezSampieri et al, 2014; Nuñez, 2017; Teddle \& Tashakkori, 2010). No es hasta finales del siglo pasado, cuando el Enfoque de MMI comienza a adquirir entidad propia a raíz del afianzamiento del concepto de triangulación o comparación e integración de datos de distinta naturaleza, métodos, investigadores, teorías y/o disciplinas (Hernández-Sampieri, 2014, p.25), sobre el que se irá desarrollando la discusión científico-metodológica en las siguientes décadas (Núñez, 2017). A partir de los años cincuenta, comienza así a consolidarse emergiendo lo que podríamos considerar la 
primera de las cuatro etapas principales que pueden distinguirse en su evolución (Cameron, 2014). Esta primera etapa o período formativo (años 50-80) va seguida respectivamente de un debate paradigmático (segunda etapa entre los años 70-90), y de un desarrollo procedimental que comienza en los años 80. Es ya a partir del presente signo (año 2000), cuando asistimos a una cuarta y última etapa en la que el MMI se separa del diseño para afianzarse como un enfoque propio de Investigación, definido en los siguientes términos:

Un enfoque de indagación que implica la recolección tanto de datos cuantitativos como cualitativos integrando ambos y empleando distintos diseños que conllevan determinadas asunciones filosóficas y marcos teóricos. La asunción esencial de esta forma de investigación es que la combinación de enfoques cualitativos y cuantitativos, proporciona una mayor comprensión del problema de investigación que empleando cada uno de ellos por separado (Creswell, 2014, p. 31)

Se dota así a dicho enfoque de entidad propia diferenciándose tanto del cuantitativo como del cualitativo, en sus distintos elementos configuradores (Tabla 1):

Tabla 1

Principales elementos en los principales enfoques de investigación.

\begin{tabular}{|c|c|c|c|}
\hline & ENFOQUE CUALITATIVO & ENFOQUE CUANTITATIVO & ENFOQUE MÉTODOS MIXTOS \\
\hline $\begin{array}{l}\text { Supuestos } \\
\text { filosóficos }\end{array}$ & $\begin{array}{l}\text { Constructivista } \\
\text { De apoyo } \\
\text { Participativas }\end{array}$ & Post-positivista & Pragmático \\
\hline $\begin{array}{l}\text { Estrategias } \\
\text { de indagación }\end{array}$ & $\begin{array}{c}\text { Fenomenología } \\
\text { Teoría Fundamentada } \\
\text { Etnografía } \\
\text { Estudio de caso } \\
\text { Narrativa }\end{array}$ & $\begin{array}{l}\text { Experimentales } \\
\text { No } \\
\text { Experimentales (Encuestas) }\end{array}$ & $\begin{array}{l}\text { Secuencial } \\
\text { Concurrente } \\
\text { Transformador }\end{array}$ \\
\hline Métodos & $\begin{array}{l}\text { Preguntas abiertas } \\
\text { Enfoques emergentes }\end{array}$ & $\begin{array}{l}\text { Preguntas cerradas } \\
\text { Enfoques predeterminados }\end{array}$ & $\begin{array}{l}\text { Preguntas abiertas y cerradas } \\
\text { Enfoques emergentes y } \\
\text { predeterminados }\end{array}$ \\
\hline Datos & Texto e imagen & Numéricos & $\begin{array}{l}\text { Datos numéricos } \\
\text { Texto e imágenes }\end{array}$ \\
\hline $\begin{array}{l}\text { Rol del } \\
\text { investigador/a }\end{array}$ & $\begin{array}{ll}\text { - } & \text { Posiciones propias } \\
\text { - } & \text { Obtiene los significados } \\
\text { de los participantes } \\
\text { - } \quad \text { Enfocado en un solo } \\
\text { concepto o fenómeno } \\
\text { - } \quad \text { Involucra sus valores } \\
\text { personales en el estudio } \\
\text { Estudia el } \\
\text { contexto/escenario de } \\
\text { los participantes }\end{array}$ & 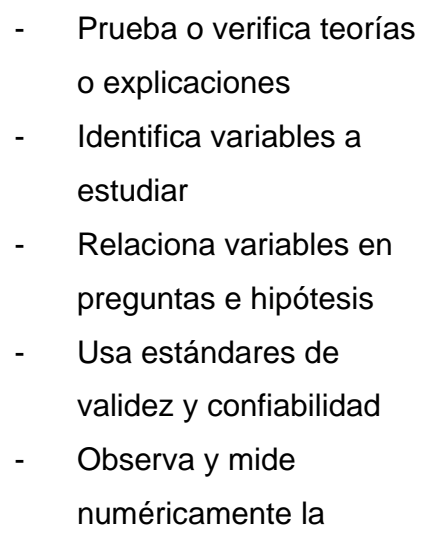 & $\begin{array}{l}\text { - } \text { Obtiene datos cuantitativos y } \\
\text { cualitativos } \\
\text { - } \quad \text { Desarrolla un razonamiento } \\
\text { para combinar } \\
\text { - } \quad \text { Integra los datos en } \\
\text { diferentes etapas de la } \\
\text { investigación } \\
\text { - Presenta imágenes visuales } \\
\text { de los procedimientos en el } \\
\text { estudio }\end{array}$ \\
\hline
\end{tabular}




$\begin{array}{llll}\text { - Valida a precisión de los } & \text { - } & \text { Unformación } & \\ \text { hallazgos } & & \text { Emparciales, objetivos } & \text { Emplea prácticas de la } \\ \text { - } & \text { Hace interpretaciones } & \text { - } & \text { Emplea procedimientos } \\ \text { de los datos } & \text { estadísticos } & \text { cuantitativa. } \\ \text { - Crea una agenda para } & & \\ \text { el cambio o reforma } & & \\ \text { Colabora con los partic. }\end{array}$

Fuente: Creswell, (2014)

Una caracterización más explícita de los distintos elementos del Enfoque de MMI, se sintetiza en la Tabla 2 a continuación:

Tabla 2

Principales elementos del Enfoque de MMI

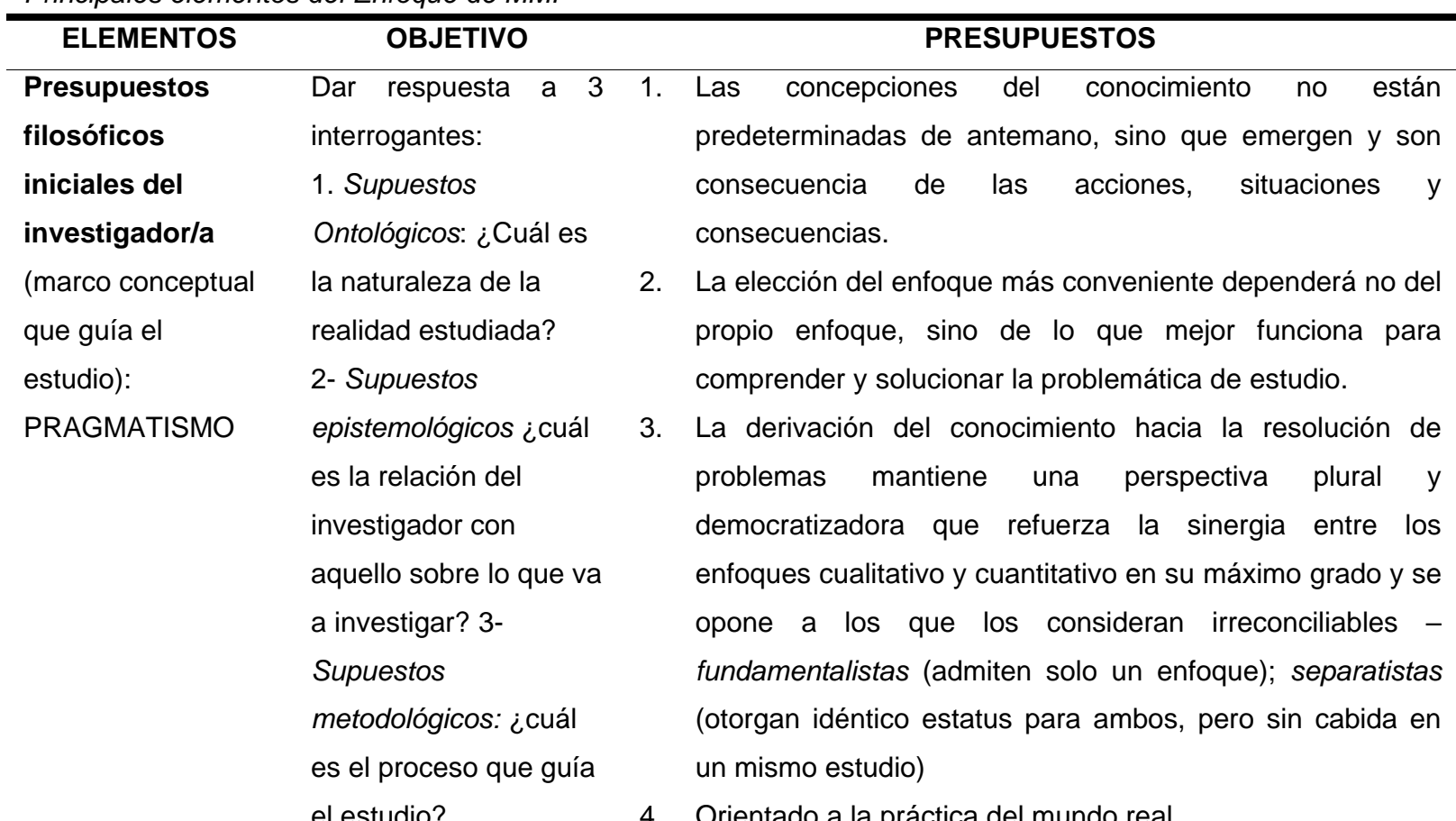

Nivel aplicado. Contribuir al desarrollo 1. Procedimientos secuenciales: para ampliar los hallazgos Procedimientos y de los MMI estrategias de un método con otro. a) Exploratorio (en la primera fase se obtienen datos cualitativos y en una segunda cuantitativos) b) Explicativo (prioridad a los datos cuantitativos en una primera fase; los datos cualitativos de la segunda fase, apoyan la interpretación de los hallazgos de los primeros).

2. Procedimientos concurrentes: convergencia en el mismo tiempo de datos cuantitativos-cualitativos para proporcionar un análisis comprensivo del problema de investigación. Se 
integra la información en los resultados generales.

3. Procedimientos transformadores: empleo de una lente teórica para proporcionar un marco para los tópicos de interés desde un procedimiento secuencial o concurrente.

Métodos

Toma de decisiones para recabar y analizar datos.
1. Métodos determinados y emergentes: permiten obtener datos ya especificados en el planteamiento del estudio e información de los participantes.

2. Preguntas abiertas y cerradas.

3. Múltiples formas de obtención de datos (cuantitativoscualitativos)

4. Análisis de texto y estadístico.

Fuente: Elaboración propia a partir de: Creswell (2014), Hernández-Sampieri (2014), y Johnson y Onwuegbuzie (2004)

Empero lo argumentado, el debate entre detractores y valores de la integración de enfoques y el esfuerzo entre éstos últimos por otorgarle la necesaria coherencia en su concepción, implementación y desarrollo, continúa sobre el tapete, como muestra el trabajo de Mertes y sus colaboradores (2016) "the Future of Mixed Methods: A Five Year Projection to 2020" publicado por la Asociación Internacional de Métodos Mixtos (MMIRA). También son diversos los autores que han advertido de las dificultades de su implementación en la práctica. Bryman (2006) o Niglas (2004) señalan entre ellas: la necesidad de integrar adecuadamente ambos enfoques; el mayor tiempo y recursos necesarios; la necesidad de justificar y argumentar ampliamente por qué y cómo es empleado el método mixto en un estudio en particular; las preferencias metodológicas del investigador; audiencias distintas; la falta de entrenamiento, conocimientos y habilidades; o los diferentes ritmos y tiempos de indagación. En general, se coincide en la conveniencia de explicitar adecuadamente el diseño y procedimiento en cualquier estudio que se lleve a cabo bajo este enfoque para mitigar posibles cuestionamientos, así como retos potenciales a los que, como investigadores e investigadoras podemos enfrentarnos (Creswell, 2014; Hernández-Sampieri et al, 2014).

\section{2- Razón de ser y finalidad del Enfoque de Métodos Mixtos de Investigación en la Investigación Educativa.}

Considerado en los términos que se acaban de exponer, se subraya la pertinencia del MMI en campos de estudio complejos y de difícil respuesta como lo es el educativo: la educación siempre ha sido un campo nuclear para la investigación de métodos mixtos (Creswell y Garrett (en De Liste, 2011, p. 95). La evidencia de su afianzamiento y proliferación en el ámbito educativo tiene un claro reflejo a nivel científico a la luz de la defensa de este enfoque tanto en múltiples congresos y publicaciones (Niglas, 2004; Bryman, 2006, Ruiz, 2008), como en la consolidación progresiva de revistas científicas especializadas ${ }^{2}$, y la aparición reciente de diversos manuales en los que se

\footnotetext{
${ }^{2}$ Journal of Methids Research; Quality and Quantity International Journal of Methodoloy, o International Journal of Multiple
} 
aborda (entre otros, Creswell, 2014; Hernández-Sampieri et al, 2014; Morse \& Niheaus, 2009, o Teddlie \& Tashakkori, 2010). Asimismo, la consolidación de la asociación internacional de investigadores sobre el particular (Mixed Methods International Research Association: MMIRA), es reseñable respecto al énfasis en el auge y la importancia actual a la que venimos refiriéndonos sobre el afianzamiento de los MMI (Cameron, 2014); Hernández-Sampieri et al., 2014 y Nuñez, 2017). 3-

Mas específicamente, se argumenta que enfoque de MMI como tercera vía de indagación en la investigación social y educativa, tiene su razón de ser en la posibilidad de integración metodológica que los caracteriza; lo que se ha dado en denominar complementariedad por deficiencia (Aravena, Kileman, Micheli, Torrealb y Zúñiga, 2006, p. 25). Esto es, permite dar respuesta a problemáticas socio-educativas que dada su complejidad no han tenido una respuesta a través de único enfoque por separado. Los MMI aportan, desde una mirada más flexible, funcional, conciliadora y enriquecida, nuevas formas de aproximación y solución (Aravena et al, 2006; Díez, 2014). En términos de Merters y sus colaboradores, tienen el potencial de contribuir a encontrar soluciones a "wicked problems" 3 dado que estimulan nuevas formas de cuestionar e implicarse en el uso de innovaciones en metodologías necesarias para abordar la complejidad. (2016, p.5).

En la misma línea, autores como Bisquerra (2004), Díaz, (2014) o Niglas (2004) defienden que es en el plano metodológico y práctico, donde sus contribuciones para el ámbito educativo son evidentes: actualmente, en el plano técnico y procedimental de la investigación educativa está ampliamente aceptada una actitud integradora con la posibilidad de utilizar diseños multimétodo ante una realidad que se presenta compleja, dinámica y plantea problemas difíciles de resolver (Bisquerra, 2004, p. 79). Y por su parte, Hernández-Sampieri et al. (2014, pp. 537-538) aluden a sus potencialidades para el estudio de fenómenos como el abandono escolar y su prevención, y otros ámbitos relacionados, haciéndose eco de, entre otros, las siguientes atribuciones vinculadas a los MMI: 1. Triangulación (incremento de la validez interna y externa del estudio); 2. Compensación (capacidad para contrarrestar las debilidades de los métodos individualmente considerados y robustecer las fortalezas de ambos); 3. Complementación (visión más comprensiva de los planteamientos de investigación y mayor entendimiento y clarificación de resultados); 4. Amplitud: visión más comprehensiva, integral y completa del fenómeno; 5. Multiplicidad: diferentes preguntas de indagación y respuestas más profundas; 6. Mayor capacidad de explicación de resultados; 7. Reducción de la incertidumbre ante resultados inesperados.

\section{METODOLOGÍA}

\section{Objetivos}

Como se ha señalado más arriba, el propósito perseguido con la Tesis aludida, fue el de contribuir a generar conocimiento sobre el contexto, finalidad y puesta en práctica del Aula 
Ocupacional en la Región de Murcia (España), durante su fase experimental en el curso escolar 2016-2017, tomando en consideración las claves políticas, institucionales, organizativas y pedagógicas que subyacen a su génesis, diseño y desarrollo, para determinar en qué medida contribuyen a lograr unos determinados resultados de aprendizaje e implicación de su alumnado. A la luz del propósito general esgrimido, dos son los objetivos en particular que se persiguen y a los que se da respuesta en el presente artículo:

1) Argumentar y justificar la pertinencia del Enfoque de Métodos Mixtos de Investigación para el estudio de fenómenos educativos de gran complejidad como lo son las problemáticas de desenganche y riesgo de abandono escolar en estudiantes que cursan programas de reenganche, en concreto, en el contexto del Aula Ocupacional.

2) Describir y analizar sobre la pertinencia y dificultades tras implementar un diseño de investigación desde el enfoque de Métodos Mixtos de Investigación para el estudio del Aula Ocupacional.

\section{Método}

Revisión bibliográfica sistemática atendiendo a los siguientes criterios de selección: 1) Documentos teóricos y de investigación de carácter nacional e internacional sobre el riesgo de exclusión educativa y social; los procesos de desenganche y abandono escolar conducentes a tales situaciones de riesgo; y puesta en práctica exitosa de programas y medidas para revertir tales problemáticas esencialmente en contextos escolares. 2) Normativa vinculante tanto a nivel europeo, nacional (España) y autonómico (Comunidad Autónoma de la Región de Murcia) en materia de abandono escolar, medidas de atención a la diversidad, así como programas específicamente orientados a paliar desenganche y evitar absentismo y abandono escolar. 3) Documentos relativos al Enfoque de Métodos Mixtos, tanto de carácter teórico, como aquellos que evidencian su pertinencia y justificación en la investigación educativa y más específicamente, en el ámbito de estudio referido.

La revisión llevada a cabo desde el punto de vista teórico, legislativo y metodológico responde a la aplicación rigurosa del método científico que permite al investigador la obtención relevante y profundización en el objeto de estudio (Bisquerra, 2016), así como, una familiarización de la temática a analizar y su estructuración en los distintos ejes sobre los que pivota (Pacios, 2013). Mediante un muestreo no probabilístico intencional se seleccionaron los documentos de forma deductiva durante el período (2010-2017) a través de las bases de datos BOE.es; BORM.es: Carm.es; Dialnet; ERIC, EUR-lex Europa.eu; Eurydice, INEE; Latindex; OCDE Education Database; Educación Database, Sccielo, Scopus, Web of Sience; conformando un corpus documental de un total de 448 documentos. La información se analizó cualitativamente a través de la técnica de análisis de contenido (Miles, Huberman \& Saldaña, 2014) mediante la delimitación de unidades de análisis, codificación abierta y categorización, con el apoyo del software Atlas ti V8. 


\section{RESULTADOS Y DISCUSIÓN}

\section{1.- Para situarnos: breve caracterización normativa del Aula Ocupacional}

El Aula Ocupacional es uno de los tres programas de re-enganche desarrollados en los centros escolares de la Región de Murcia (España) -junto con el PAI (Programa de Aprendizaje Integral) y la Formación Profesional Básica (FPB)-, que cuenta con un amplio desarrollo legislativo, analizado detenidamente en el contexto del proyecto de investigación más amplio. Nos referiremos aquí únicamente y de forma gráfica (Tabla 3, más abajo), con el fin de situar y contextualizar la realidad objeto de estudio, a algunos de sus rasgos y características más definitorias de la legislación vigente en el momento en el que fue analizada ${ }^{4}$.

\section{Tabla 3}

Finalidad y principales características de las Aulas Ocupacionales en la Región de Murcia

\begin{tabular}{|c|c|}
\hline Finalidad & $\begin{array}{l}\text { Reducir el absentismo y el riesgo de abandono escolar temprano dotando al alumnado de las } \\
\text { competencias personales, sociales y profesionales que favorezcan: a) su continuidad en el } \\
\text { sistema educativo, preferentemente en un ciclo formativo de Formación Profesional Básica o } \\
\text { en un Programa Formativo Profesional de modalidad adaptada. b) Su inserción socio-laboral } \\
\text { y su incorporación a la vida activa con responsabilidad y autonomía. }\end{array}$ \\
\hline Destinatarios & $\begin{array}{l}\text { Alumnado que tenga } 15 \text { años o los cumpla en el año de acceso a esta medida y deba cursar } \\
2^{\circ} \text { o } 3^{\circ} \text { curso de la ESO durante su año de acceso, que no esté en condiciones de } \\
\text { promocionar, haya repetido algún curso de la ESO, y tenga abierto expediente de } \\
\text { absentismo. Se priorizan los estudiantes con desfase curricular en el bloque de asignaturas } \\
\text { troncales y con conductas contrarias a las normas de convivencia en el centro durante el } \\
\text { curso anterior a su acceso. }\end{array}$ \\
\hline Estructura & $\begin{array}{l}\text { Modular: } \\
\text { a) Módulos asociados a unidades de competencia profesional. } \\
\text { b) Módulos y ámbitos generales no asociados a unidades de competencia profesional: } \\
\text { Ámbito de Ciencias Aplicadas. Ámbito Sociolingüístico. Dos módulos optativos. } \\
\text { Módulo de Formación en centros de Trabajo (FCT). }\end{array}$ \\
\hline Ratio & 8-12 estudiantes (e un solo grupo) \\
\hline Duración & Un curso escolar \\
\hline Certificación & $\begin{array}{l}\text { Certificación académica de los módulos y ámbitos cursados y las unidades de competencia } \\
\text { profesional acreditadas. La superación de éstas últimas tendrá carácter acumulable para su } \\
\text { reconocimiento en estudios posteriores. }\end{array}$ \\
\hline Titulación & No titula \\
\hline Autorización & $\begin{array}{l}\text { De las } 9 \text { Aulas vigentes en el curso académico anterior que son autorizadas con carácter } \\
\text { provisional. }\end{array}$ \\
\hline
\end{tabular}

Fuente: Resolución de 27 de julio de 2016, de la Dirección General de Innovación Educativa y Atención a la Diversidad, por la que se dictan instrucciones para la adaptación, con carácter experimental en el curso 2016-

\footnotetext{
${ }^{4}$ Cabe señalar que el Aula Ocupacional fue analizada en un momento de transición legislativa, la normativa de 2016 introdujo cambios sustanciales respecto a la legislación anterior, y posteriormente ha sido también modificada incluyendo ciertas variaciones o modificaciones estructurales.
} 
2017, de las aulas ocupacionales existentes durante el curso 2015-2016.

Se puede apreciar a tenor de lo contemplado en la tabla anterior, que se trata de un programa pensado para un alumnado con altos niveles de problematicidad ligados estrechamente a una conducta negativa, absentismo y desenganche escolar. La finalidad es proporcionar a este alumnado una formación de carácter profesional elemental, complementada con otra más académica, ligada a materias que se imparten en la ESO, que se agrupan en ámbitos de conocimiento (con menos fragmentación curricular que en la ESO regular). Se desarrolla en instalaciones municipales, fuera de los Institutos, aunque vinculada administrativamente a uno de ellos. Una vez cursada esta medida y finalizada con éxito, no se prioriza regresar a la ESO ordinaria: los alumnos podrían continuar preferentemente cursando un programa de FPB si cumplen los requisitos de acceso, o un Programa Formativo Profesional adaptado (versión adaptada del FPB, generalmente en asociaciones sin ánimo de lucro u ONGs).

\section{2- Propuesta metodológica de un diseño de MMI para el estudio del Aula Ocupacional}

Cuando nos situamos en el enfoque de MMI, el diseño de investigación o, estrategia concebida para obtener la información que se requiere, dar respuesta al problema formulado y cubrir los intereses del estudio (Bisquerra, 2004, p. 120) tiene sus propias particularidades. En concreto, aquellas que conciernen al correspondiente al estudio de las Aulas Ocupacionales, y que pueden ser tenidas en consideración para el análisis de otros programas de re-enganche, se contemplan en la tabla 4 más abajo.

La justificación y adecuación de la propuesta del diseño denominado DIACNIV: Diseño Anidado Convergente Multinivel de Método Dominante Cualitativo radica, como se viene argumentando, en la propia naturaleza el problema de investigación. El estudio de programas de reenganche como el Aula Ocupacional, requieren de su comprensión tal y como son significados por sus participantes.

Así y, en primer lugar, el diseño DIACNIV, permite adecuarse a la perspectiva teórica que guía el estudio, siendo en la investigación sobre el Aula Ocupacional el modelo ecológico de contextos, factores y dinámicas del riesgo de exclusión educativa de (Martínez, Escudero, González y otros, 2004), y el modelo multidimensional de implicación escolar de Fredericks et al (2004). En relación a tales planteamientos teóricos de partida, así como al propósito de la investigación, un análisis deductivo de la información cuantitativa, dejaría de lado otras muchas cuestiones y matices

sin las que no es posible comprender la idiosincrasia de lo que acontece en la práctica real y diaria, y las dinámicas de funcionamiento cotidianas que se suceden en dichas Aulas. En aras a su comprensión, el diseño DIACNIV permite posicionarnos en la Fenomenología como enfoque prioritario que guía la investigación en tanto que permite llevar a cabo la inducción derivada de aquellas particularidades contextuales a las que no se puede llegar sino de forma flexible y subjetiva. En términos generales y, en particular, en el ámbito educativo, ofrece la confianza suficiente para 
adentrarse en el mundo de los sujetos e identificar cómo y bajo qué condiciones trabajan o viven, así como definir el significado que le dan a una experiencia a partir de su propia visión (Creswell, 2015, Hernández-Sampieri et al, 2014). Asimismo, y aunque los fundamentos fenomenológicos son los mayoritarios se deja abierta una vía inductiva para el descubrimiento a través de las aportaciones de los diversos participantes desde un plano subjetivo desde el que pueden emerger nuevas contribuciones sobre el problema de investigación. Pero, además, su naturaleza científica ofrece áreas de compatibilidad metodológica con enfoques más deductivos que refuerzan el estudio (Mayoh \& Onwuegbuzie, en Kanga, Njeru, Wachera, \& Ryutere, 2015): la incrustación del método descriptivo en el mencionado enfoque mayoritario permite especificar propiedades y características importantes de cualquier fenómeno que se analice y mostrar con precisión sus ángulos o dimensiones y las tendencias del grupo estudiado (Hernández-Sampiri et al, 2014).

Tabla 4

Caracterización del Diseño Anidado Convergente Multinivel de Método Dominante Cualitativo (DIACNIV), en el estudio del Aula Ocupacional

DISEÑO ANIDADO CONVERGENTE MULTINIVEL DE MÉTODO DOMINANTE CUALITATIVO (DIACNIV)

Variante del En una sola fase en la que se lleva a cabo de forma simultánea la recogida de la diseño información tanto cualitativa como cuantitativa.

Concurrente o

Convergente

Método La investigación se guía por la Fenomenología (método cualitativo) y el método dominante: Descriptivo (cuantitativo) de menor peso, se inserta o anida en el primero.

cualitativo

Diseño multinivel Variante del diseño anidado que emplea metodologías cuantitativa y cualitativa para buscar información en diferentes niveles o en grupos de análisis.

Integración

Relación significativa entre las ramas cualitativas y cuantitativa: en el mismo estudio se recolectan, analizan interpretan y se informa de los resultados de ambos tipos de datos de forma mutuamente iluminadora.

Puede llevarse a cabo en distintos planos: en la recogida de datos (en una sola fase); en el análisis de los datos (mediante la transformación de la información cualitativa o códigos en cuantitativa o números) y en la interpretación conjunta de los datos.

Fuente: elaboración propia a partir de Creswell (2014), Onwegbuzie \& Combs, (2011) y Tashakkori \& Teddlie (2010).

En cuanto a la variante "multinivel" del diseño aludido, está especialmente indicada para el estudio de organizaciones y aspectos organizativos de una determinada institución: los diferentes colectivos extraídos de un mismo universo proporcionan la visión de los distintos niveles de interacción con los aspectos o variables de interés y con ello, una mayor comprensión del problema 
de investigación (Hernández-Sampieri et al, 2014). Esto ha permitido comprender y adentrarnos en la interrelación entre los distintos elementos y condicionantes que intervienen en el desarrollo del Aula Ocupacional y que influyen en sus resultados en distintos planos o niveles: macro o políticas, meso o nivel del centro escolar, y micro o aula.

Finalmente, la integración como aspecto consustancial al enfoque de MMI, se centra, en el diseño propuesto, en la recogida e interpretación de los datos (metainferencias), en correspondencia con la perspectiva más general del diseño concurrente. De igual modo, ambas vías de integración estarían de acuerdo con los planteamientos del diseño anidado multinivel en el que los análisis de los datos pueden variar en todos los niveles estudiados, aunque sí es preciso integrar de forma conjunta los resultados en todos ellos (Tashakkori \& Teddlie, 2010). Atendiendo a las consideraciones anteriores, podemos decir de un lado que se dota de fortaleza a este diseño en particular. Pero, de otro, no podemos obviar que también se dificulta en diversos sentidos. A saber:

a) En relación a la concurrencia temporal en la recogida de información, lo que implica prestar atención a las particularidades de los procedimientos cualitativo-cuantitativo en una sola fase y en distintos niveles.

b) La complejidad existente en su fase de interpretación en la que se ha de lograr la significación precisa entre ambos procedimientos (cualitativo-cuantitativo)

c) La escasez de procedimientos con pautas precisas y delimitadas para guiar el proceso (Onwegbuzie \& Combs, 2011; Onwegbuzie \& Leech, 2006).

d) Una mayor dificultad a la hora de garantizar la calidad y la validez en todas las etapas de su desarrollo. En este último aspecto, las tendencias más recientes incorporan elementos propios para garantizar la calidad del diseño al que nos referimos, en relación a tres criterios esenciales:

a. Calidad en el diseño: en relación al uso e implementación efectiva de los procedimientos más adecuados para responder al problema de investigación. 1) Adecuación del diseño al propósito del estudio. 2) Consistencia interna entre sus componentes. Y, 3) implementación fiel a la práctica acorde con los procesos de selección de la población objeto de estudio, recogida y análisis de datos mixtos.

b. Rigor interpretativo o inferencias de calidad: congruencia entre las propias inferencias, y respecto a la teoría y conocimiento de base del estudio, acuerdo interpretativo, y transferencia de inferencias entre la población participante.

c. Legitimidad, En relación a: 1) la integración de la muestra de modo que se permita la contribución al logro de metainferencias reflejando la visión complementaria y subjetiva del MMI. 2) legitimidad interna-externa (de los participantes e investigador/es); 3) 
consideración de las debilidades potenciales

En suma, y aún teniendo en cuenta las dificultades que se acaban de describir, se trata de un diseño adecuado para describir una situación educativa y/o clasificarla en una categoría determinada (Martínez, 2007, p. 31) y con gran aceptación en el campo educativo (empleado en el $65 \%$ de las investigaciones según documentaba Niglas, ya en 2004), a la hora de determinar qué aspectos del problema son significativos y qué dimensiones del mismo tienen relación entre sí (Martí, Bo y Climent, 2010).

De forma gráfica, el diseño referido se puede apreciar en la figura 1 siguiente:

\section{Figura 1}

Diseño anidado convergente multinivel de método dominante cualitativo (DIACNIV) ${ }^{5}$ de la investigación sobre Aula Ocupacional en la Región de Murcia.

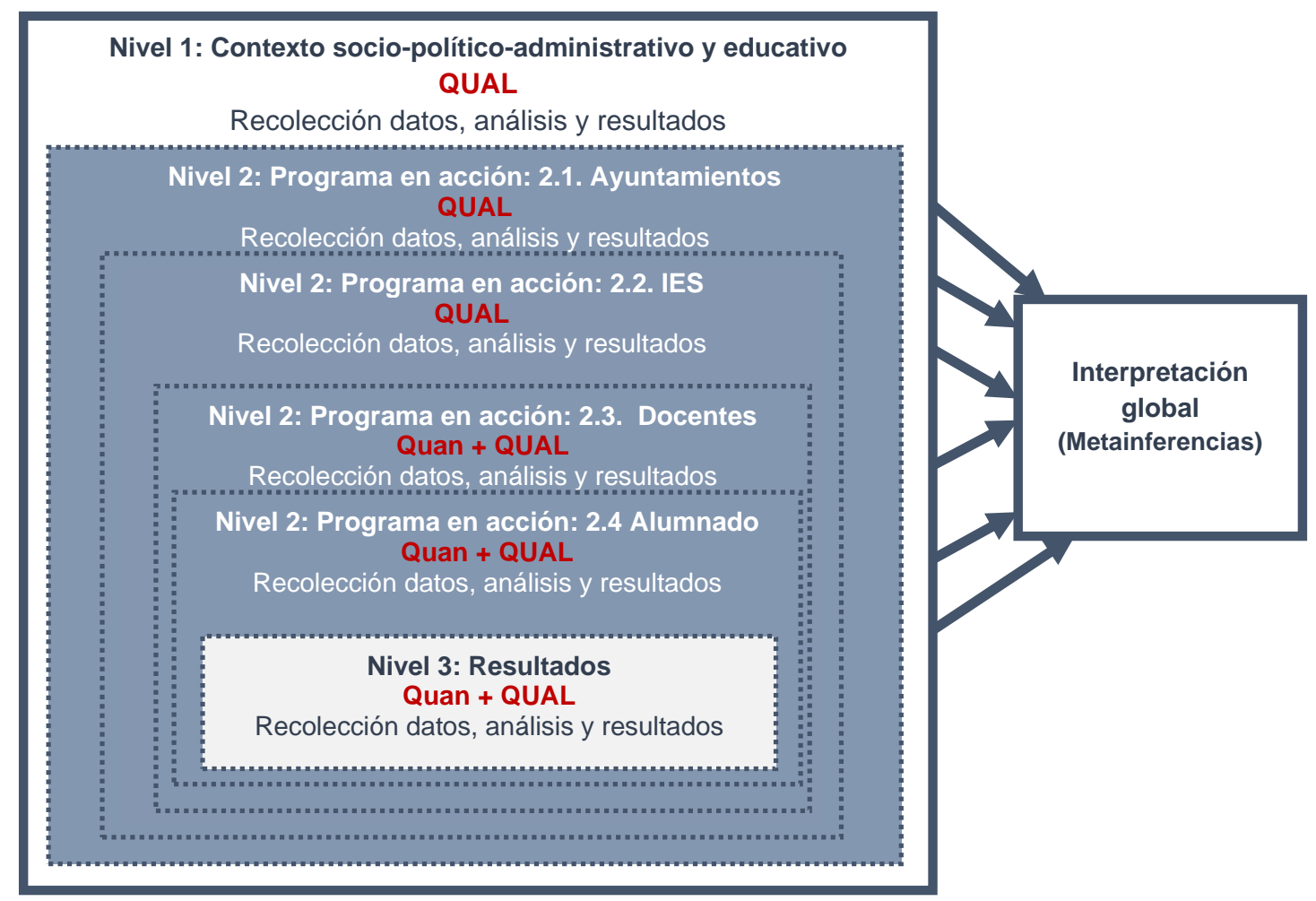

Elaboración propia en base al modelo de Hernández-Sampieri et al (2014).

\footnotetext{
${ }^{5}$ La notación del diseño presentado sigue la nomenclatura establecida en su momento por Morse (1991) tal y como se recoge en Creswell (2014) en relación a las siguientes consideraciones:

"+" indica una forma simultánea o concurrente de obtención de datos.

El uso de mayúsculas indica un énfasis o prioridad en los datos cualitativos y su análisis en el estudio.

"CUAN" y "CUAL" representan cuantitativo y cualitativo, respectivamente.

Bajo cada figura se especifican los procedimientos de obtención, análisis e interpretación de datos para ayudar al lector a comprender los procedimientos más específicos que se utilizan.

Los recuadros destacan la obtención de datos cuantitativos y cualitativos.
} 


\section{CONCLUSIONES}

En consonancia con lo relatado en los apartados que preceden, se considera y defiende que, las contribuciones a la generación del conocimiento sobre el Aula Ocupacional en todos los aspectos pretendidos, fue posible gracias al planteamiento adoptado y defendido sobre riesgo escolar y desde una concepción multidimensional (conductual, cognitiva y afectiva) del enganche y el desenganche escolar. Y, en consonancia con el mismo, la problemática de estudio y los objetivos de investigación, al situarnos, desde el punto de vista metodológico, en un enfoque y un diseño que han permitido conjugar e integrar la información cuantitativa y cualitativa recabada y analizada, para obtener una panorámica global y ajustada a la realidad de estas Aulas en sus múltiples planos de análisis. Asimismo, profundizar en su comprensión dando voz a los directamente implicados. Las propias visiones, percepciones y valoraciones de los distintos informantes entre las que cabe reseñar las vivencias de estudiantes y docentes en el programa, fueron claves para poder sacar a la luz determinados aspectos sobre su funcionamiento y resultados que, permaneciendo en un plano más profundo han estado largo tiempo ocultos siendo invisibilizados.

En suma, solo conducidos por dispositivos metodológicos que no han sido tradicionalmente los más afluentes en un campo de investigación protagonizado por estudios a gran escala y que, sin restar la importancia que merecen, han dejado de lado otros razonamientos de carácter subjetivo, las repercusiones de la organización escolar, o la responsabilidad institucional, fue posible mostrar la realidad y repercusiones del programa estudiado. Así y dejando constancia de su complejidad y dificultades a la hora de llevarlo a la práctica, consideramos que el MMI no solo es útil y necesario, sino que además ofrece la validez y el rigor necesarios para avanzar científicamente en la investigación educativa, y es especialmente indicado para el análisis de programas de reenganche como el Aula Ocupacional. Finalmente, y desde el punto de vista personal, ofrece satisfacciones difícilmente alcanzables con otras opciones metodológicas.

\section{REFERENCIAS}

Aravena, M., Kileman, E., Micheli, B., Torrealba, R. y Zúñiga, J. (2006). Investigación Educativa I.: Universidad Arcis,

Bisquerra (coord.) (2004). Metodología de la Investigación Educativa. La Muralla.

Bisquerra, R. (2016). Metodología de la investigación educativa. La Muralla

Bolívar, A. y López, L. (2009). Las grandes cifras del fracaso y los riesgos de exclusión educativa. En Profesorado, Revista de Currículum y Profesorado, 13 (3), 51-78.

Bryman, A. (2006). Integrating quantitative and qualitative research: how is it done? Qualitative Research, 6 (1), 97-113.

Cameron, R. (2014). Mixed Methods Research Workshop. Co-Convenor of Mixed Methods SIG ANZAM Research Fellow. Curtin Business School, Deakin University Melbourne, 2 july.

Choi, A. y Calero, J. (2013). Determinantes del riesgo del fracaso escolar en España PISA-2009 y propuestas de reforma. Revista de educación, 362, 562-593.

Christenson, S. L., Reschly, A. L., \& Wylie, C. (eds) (2012). The handbook of research on student 
engagement. Springer Science.

Comisión Europea (2011): Abordar el abandono escolar prematuro: una contribución clave en la agenda 2020. Comunicación de la Comisión al parlamento europeo, al consejo, al comité económico y social europeo y al comité de las regiones. (COM (2011) 18 final). Bruselas, 2011, 31 enero.

Creswell, J.W. (2014). Research design: Qualitative, Quantitative and mixed approaches (4를. ${ }^{-}$.). Sage.

Day, L. (dir.) (2013). Preventing Early School Leaving in Europe-Lessons Learned from Second Chance Education. European Comission.

De Liste, J. (2011). The beneficts and Challenges of Mising Methods and Methodologies: Lessons Learnt From Implementing Quatlitatively Led Mixed Methods Research Designs in Trinidad and Tobago. Caribbean Curriculum, 18, 87-120.

Díaz, S.M. (2014). Los métodos mixtos de Investigación: presupuestos generales para la evaluación educativa. Revista Portuguesa de Pedagogía, 48-1, 7-23.

Escudero, J.M. y Martínez, B. (2012). Las políticas de lucha contra el fracaso escolar: ¿programas especiales o cambios profundos del sistema y la educación? Revista de Educación, número extraordinario, 174-193.doi: 10.4438/1988-592X-RE-2012-EXT-211

European Commission (2015a). A whole aproach to tackling early school leaving Policy messages. European Union. DG Education and Culture.

Faci, F.M. (2011). El abandono escolar prematuro en España. Avances en Supervisión Educativa, 14, $1-26$.

Fernández, E.; Muñoz de Bustillo, R.; Braña, F.J. y Antón, J.I. (2010). Algunas apreciaciones aritméticas sobre el fracaso y el abandono escolar en España. Revista de Educación, $n^{-}$ extraordinario, 307-324

Fredericks, J.A.; Blumenfied, P.C. y Paris, H. (2004). School engagement: Potential of the concept. State of evidencie. Review of Educational Research, 74 (1), 59-109

GHT Consluting Ltd; Nevala, A.M. y Hawley, J. (2011). La reducción del abandono escolar en la Unión Europea. Dirección general de políticas interiores de la Unión. Departamento temático B: políticas estructurales y de cohesión. Parlamento Europeo.

González, M. T. (2015). Los centros escolares y su contribución a paliar el desenganche y el abandono escolar. Profesorado, Revista de currículum y formación del profesorado, 19 (3), 158-176. doi. https://doi.org/10.30827/recfpro.v19i3.43639

Harris, L. (2011). Secondary teachers 'conceptions of student engagement: Engagement in learning or in schooling? Teaching \& Teacher Education, 27, 373-386.

Hernández-Sampieri, R., Fernández, C. y Baptista, P. (2014). Metodología de la investigación (6 $6^{\underline{a}}$ Ed.). McGraw Hill Education.

Kanga, A., Njeru, L.N., Wachera, E. \& Rutere, J. (2015). Rethinking Variant Models of Embedded Research design within a qualitative dominant Mixed Method study. General Education Journal, 4 (2), 15-31.

Lara García, B. González-Palacios, A.; González Álvarez y Martínez-González, M.G. (2014). Fracaso 
escolar: conceptualización y perspectivas de estudio. Revista de Educación y Desarrollo 30 , 71-83.

Marcelo, E.; Araújo, H.; Magalhães, A y Rocha, C. (2015). La construcción del abandono temprano de la escuela como concepto político: un análisis en la sociología de la educación. Profesorado, Revista de currículum y formación del profesorado, 19 (3), 28-42.

Marhuenda, F y García, J. (2017). la educación de la juventud: ¿es posible superar los límites de la educación obligatoria? Profesorado, Revista de currículum y formación del profesorado, 21 (4). 1-15

Martí, R., Bo, M.R. y Climent, C.I. (2010). Propuesta de Análisis de Contenido Fenomenológico de los Datod Obtenidos en la Entrevita. UT. Revista de Ciènces de IÉducació, juny, 113-133.

Martos, J.M. (2016). Estudiantes en riesgo de exclusión educativa en la Enseñanza Secundaria Obligatoria de Andalucía: hacia una comprensión desde el discurso político y del profesorado. (Tesis Doctoral). Universidad de Granada.

Mertens, D., Bazeley, P., Bowleg, L., Fielding, N., Maxwell, J., Molina-Azorín, J.F. \& Niglas, K. (2016). Expanding Thinking Through a Kaleidoscopic Look Into the Future: Implications of the Mixed Methods International Research Association's Task Force Reporto $\mathrm{n}$ the Future of Mixed Methods. Journal of Mixed Methods Research, 1-7. doi: 10.1177/1558689816649719

Miles, M. B., Huberman, A.M. \& Saldaña, J. (2014). Qualitative Data Analysis. A Methos Sourcebook (3르. Ed.). Sage.

Morse, J.M. \& Niehaus, L. (2009). Mixed Method Design: Principles and Procedures. Routledge.

NESSE (Network of Expert in Social Sciences of Education and Training) (2010). Early school leaving. Lesson from reseach for policy makers. DG Education and Culture: European Commission.

NESSE (Network of Expert in Social Sciences of Education and Training) (2011). Multi/ Interdisciplinary teams for early school leaving prevention: Developing a European Strategy informed by international evidence and reseach. Research Paper for European Commission Network of Expert on the Social Research

Niglas, K. (2004). The combined use of Qualitative and Quantitative Methods in Educational Research. Trükitud.

Núñez, J. (2017). Los Métodos Mixtos en la Investigación en Educación: Hacia un uso reflexivo. Cadernos de Pesquisa, 47(164), 632-649.

OCDE (2016). Estudiantes de bajo rendimiento. Por qué se quedan atrás y cómo ayudarles a tener éxito. Resultados principales. Recuperado de http://www.oecd.org/pisa/keyfindings/PISA2012-Estudiantes-de-bajo-rendimiento.pdf

Onwuegbuzie, A.J. \& Combs, J.P. (2011). Data Analysis in Mixed Research: A Primer. International Journal of Education, 3 (1), 1-23.

Onwuegbuzie, A.J. \& Leech, N.L. (2006). Linking Research Question to Mixed Methods Martínez, R.A. (2007). La investigación en la práctica educativa: Guía Metodológica de investigación para el diagnóstico y evaluación en los centros docentes. Colección Investigamos, 끈. Ministerio de Educación y Ciencia.

Pacios, A. (2013). Técnicas de búsqueda y uso de la información. Editorial Universitaria Ramón 


\section{Areces}

Pradós, M; Duque, E. y Molina, S, (2011). Aportaciones de la Investigación Europea INCLUD-ED para a reducción del Abandono Escolar Prematuro. Avances en Supervisión Educativa, 14, 1-17.

Rambla, X. y Fontdevilla, C: (2015). Una oportunidad para evaluar las teorías del cambio: la estrategia europea contra el abandono escolar prematuro. Profesorado. Revista de currículum y profesorado), 19 (3), 43-57

Rujas, J. (2017). Dispositivos institucionales y gestión del fracaso escolar: las paradojas de la atención a la diversidad en la ESO. Cuadernos de Relaciones Laborales, 35 (2), 327-345. doi. http://dx.doi.org/10.5209/CRLA.56776

Rumberger, R. W \& Rotermund, S. (2012). The Relationship Between Engagement and High School Dropout. S. L. Christenson, A. L. Reschly, \& C. Wylie (Eds.), Handbook of research on student engagement (pp. 491-515. Springer Scienc.

Symonds, J. \& Hargreaves, L. (2016). Emotional and Motivational Engagement at Shool Transition: A Qualitative Stage-Enviroment Fit Study. Journal of Early Adolescence, 36 (1), 54-85.

Teddlie, C. and A. Tashakkori (2010). Overview of contemporary issues in mixed methods research. In A., Tashakkori, \& Teddlie, C. (Eds), Handbook of Mixed Methods in Social \& Behavioral Research (pp 1-41). Sage

Tomaszewska-Pękała, H., Wrona, A. \& Marchlik, P., (2017). Finding inspiring practices on how to prevent ESL and school disengagement. Lessons from the educational trajectories of youth at risk from nine EU countries. Publication 6. Project RESL. eu. financiado por la Unión Europea (320223).

Vaquero, A. (2011). La reducción del fracaso escolar. Asignatura pendiente del sistema educativo español. Avances en Supervisión Educativa, 1-18. 